\title{
Multidrug-Resistant Tuberculosis: Case Reports Study in a Central State of Brazil
}

\author{
João Alves de Araújo-Filho ${ }^{1,2}$, Arioldo Carvalho Vasconcelos-Jr ${ }^{1}$, Eduardo Martins de Sousa ${ }^{1}$, Colombina da Silveira ${ }^{2}$, Patrícia \\ Tavares Pereira de Sousa ${ }^{2}$, Klaus Andrade Severo ${ }^{2}$, Ludmila Ferreira Vieira ${ }^{2}$, André Kipnis ${ }^{1}$ and Ana Paula Junqueira-Kipnis ${ }^{1}$ \\ ${ }^{1}$ Institute of Tropical Pathology and Public Health of Federal University of Goiás; ${ }^{2}$ Dr. Anuar Auad Tropical Diseases Hospital; Goiânia, GO, Brazil
}

\begin{abstract}
Multidrug-resistant tuberculosis (MDR-TB) is an emerging and worrisome health problem that threatens tuberculosis (TB) control worldwide. The clinical management of MDR-TB is a complex issue associated with the use of multiple drugs for a long period, usually accompanied by side effects and high costs. The objective of this work was to relate cases of MDR-TB occurring in Goiás, a central state of Brazil. We related five cases of MDR-TB, three women and two men. All were pulmonary cases. Three were in their second treatment and two in their first treatment. Surgical pulmonary resection was performed in one case. One death occurred. Lack of adherence, gastric intolerance to antiTB drugs and poor clinical management were the main aspects related to the emergent resistance. A revision of the main clinical aspects of this disease was performed.
\end{abstract}

Key-Words: Tuberculosis, multidrug-resistant tuberculosis, MDR-TB, Mycobacterium tuberculosis.

Tuberculosis (TB) remains a major world health problem. Around two billion people are infected by Mycobacterium tuberculosis, the causal agent of this disease [1-3]. This is a third of the total world population and it is expected that nine million people will become infected each year. TB also contributes to two million deaths per year. Additionally, the emergence of multidrug-resistant tuberculosis (MDR-TB), particularly in the 1990s, has become an important health problem and threatens TB control worldwide [4,5].

In order to be considered MDR-TB, the M. tuberculosis strain should be resistant to at least rifampicin $(\mathrm{R})$ and izoniazid $(\mathrm{H})$ according to the World Health Organization (WHO) [5]. The Brazilian Ministry of Health adopts a different definition of multiresistance; resistance to $\mathrm{R}, \mathrm{H}$, and to a third drug should be present. Brazilian individuals that fail to cure with the first line regimen are treated with a standardized second line regimen that includes streptomycin (S), ethambutol (E), ethionamide (ET), and pyrazinamide $(\mathrm{Z})$. Failure during this second line regimen, associated to resistance to $\mathrm{R}$ and $\mathrm{H}$, is also considered MDR-TB [6].

The Stop TB partnership estimates that around 458 thousand MDR-TB cases must exist. Alarming also was the Centers for Diseases Control and Prevention (CDC) and WHO conclusion that $20 \%$ of the 17.690 strains analyzed worldwide between the years 2000 and 2004 were MDR, and 2\% were extensively drugresistant (XDR-TB), that is, resistant to $\mathrm{R}, \mathrm{H}$, and resistant to a fluoroquinolone and to one or more of the following injectable drugs: amikacin (AM), capreomycin, kanamycin [7,8].

Despite Brazil being among the 22 countries with $80 \%$ of all global cases of TB (about 100.000 new cases/year), MDRTB does not seem to be a major concern, with 2,372 cases between October 1995 and August 2006 [3,5,6]. These TB

Received on 11 September 2007; revised 18 January 2008.

Address for correspondence: Dr. João Alves de Araújo Filho. Instituto de Patologia Tropical e Saúde Pública da Universidade Federal de Goiás. Rua Delenda Rezende de Melo, S/N ${ }^{\circ}$, Setor Universitário, Goiânia, GO, Brazil. Zip code: 74605-050. Phone: 5562 3209-6126. Fax: 5562 3521-1839. E-mail: araujofilho63@click21.com.br.

The Brazilian Journal of Infectious Diseases

2008;12(1):94-98 (C) 2008 by The Brazilian Journal of Infectious Diseases and Contexto Publishing. All rights reserved. patients require a special treatment with second line drugs for long periods of time, associated or not with surgical interventions, usually associated with collateral damages and poorer outcome [9-11], and represent an additional burden to the local health system $[12,13]$.

Here we report and discuss five cases of MDR-TB occurring in Goiás, a central state of Brazil.

\section{Material and Methods}

The clinical charts of five patients that met the WHO and Brazilian Ministry of Health definitions of MDR-TB case in care at the Tropical Diseases Hospital Dr. Anuar Auad, Goiânia, Goiás, Brazil; a regional center for treatment of infectious diseases, were reviewed between January 1999 and May 2007. Participation was voluntary after signing the informed consent that was approved by an ethical committee.

\section{Cases Reports}

Clinical and Laboratory Characteristics of MDR-TB Patients

The clinical and laboratory characteristics of the MDRTB individuals are presented in Table 1. Resistance to three drugs was seen in three patients (\# 1,2 and 5) and to four drugs was seen in two patients (\# 3 and 4). In addition to the resistance to $\mathrm{R}$ and $\mathrm{H}$, we found resistance to $\mathrm{S}$ (\# 1 and 4), E (\# 2 and 3), Z (\# 4 and 5) and ET (\# 3). No patients met the criteria for diagnosis of XDR-TB.

Poor adherence to treatment regimen, gastric intolerance to anti-tuberculosis drugs and unsatisfactory clinical management were the major factors associated with the emergence of multidrug resistance for patients 1,2 and 3. The drugs more frequently related to gastric intolerance were $\mathrm{Z}$ (patients 1 and 3 ) and $E$ (patients 1 and 2). Patient 2 have had intolerance to $\mathrm{R}, \mathrm{H}$ and $\mathrm{E}$. A contact with a confirmed case of MDR-TB was identified in case five, which shared the same house with a cousin, MDR-TB confirmed deceased case.

All patients have had at least one period of hospitalization. No underlying disease was found. Cough and fever were the major complains. Hemoptysis was found in patients 1 and 4 . All participants of this study were HIV negative. Only one patient had a tuberculin skin test (TST) negative while all 
Table 1. Clinical and laboratorial characteristics of the MDR-TB ${ }^{1}$ patients

\begin{tabular}{|c|c|c|c|c|c|}
\hline & Patient 1 & Patient 2 & Patient 3 & Patient 4 & Patient 5 \\
\hline Gender $^{2}$ (Age in years) & $\mathrm{F}(40)$ & $\mathrm{M}(39)$ & $M(62)$ & $\mathrm{F}(33)$ & $\mathrm{F}(39)$ \\
\hline Major symptoms & $\begin{array}{l}\text { Cough, fever, } \\
\text { hemoptysis, cachexia }\end{array}$ & Cough, fever & $\begin{array}{l}\text { Cough, fever, } \\
\text { dyspnea, hemoptysis, }\end{array}$ & $\begin{array}{l}\text { Cough, } \\
\text { chest pain, } \\
\text { hemoptysis }\end{array}$ & $\begin{array}{l}\text { Cough, } \\
\text { fever, } \\
\text { weigh lost }\end{array}$ \\
\hline Antibiotic resistance ${ }^{3}$ & $\mathrm{R}, \mathrm{H}, \mathrm{S}$ & $\mathrm{R}, \mathrm{H}, \mathrm{E}$ & $\mathrm{R}, \mathrm{H}, \mathrm{E}, \mathrm{ET}$ & $\mathrm{R}, \mathrm{H}, \mathrm{Z}, \mathrm{S}$ & $\mathrm{R}, \mathrm{H}, \mathrm{Z}$ \\
\hline $\begin{array}{l}\text { Interval diagnosis } \\
\text { (TB and MDR-TB) }\end{array}$ & 2 years & 7 years & 2 years & 6 years & 1 year \\
\hline Present treatment regimen & OF,ET,CL,Z,TR & $\begin{array}{l}\text { OF,Z,ET, } \\
\text { AM,TR }\end{array}$ & AM,OF,CL,Z,TR & OF,E,ET,TR,AM & AM,E,TR,OF \\
\hline $\mathrm{TST}(\mathrm{mm})^{4}$ & 18.0 & 0.0 & 32.0 & 10.0 & 22.0 \\
\hline
\end{tabular}

1- MDR-TB - multidrug-resistant tuberculosis. 2- M - male; F- female. 3- R- rifampicin; H- isoniazid; Z- pyrazinamide; E- ethambutol; ETethionamide; S- streptomycin; OF- ofloxacin; CL- clarithromicin; AM- amikacin; TR- terizidone. 4- Tuberculin skin test.

others had TST ranging from 10.0 to $32.0 \mathrm{~mm}$. In order to restrict the disease, patient 3 underwent a lower right lung lobectomy during his first treatment, in January 2002, as indicated by his assistant physician.

The choices of drugs for treatment were determined for each case according to drug sensibility tests and individual tolerability (Table 1). At least four drugs with certain or almost certain effectiveness were included in the treatment regimen. The drug dosage was determined by body weight (data not shown) according to WHO and/or the Brazilian Ministry of Health guidelines [5,6]. Four patients had been currently under treatment. Two of them had been receiving the first course of MDR-TB treatment and two patients had been under the second treatment. The second treatment was due to an initial failure on patient 1 and due to abandonment on patient 2 . Patient 1 initiated her first treatment three years before the second regimen. The interval between the first MDR-TB and the last treatment was five years for patients 3 and 5 .

Although patient 1 had a failure on the first course of chemotherapy, her sputum smear and culture were negative after six months of the second treatment. Individual 2, which was in his first course of therapy, have developed mild renal and auditory impairment probably due to aminoglycosides and had irregular ingestion of drugs. His smear and culture are positive until nowadays. In addition, patient 2 was homeless and had difficulties to pursue a complete medical follow-up. Patient 3, due to his non-adherent treatment and refused hospitalization, evolved to progressive deterioration of pulmonary function and a re-treatment was offered. Unfortunately, patient 3 passed away on May 2007 due to extensive pulmonary damage. On her first MDR-TB treatment, patient 4 had sputum smear and culture negatives after four months. Individual 5, which was on her second treatment, had similar evolution after five months of therapy.

\section{$\underline{\text { Radiological Findings of MDR-TB Patients }}$}

In Figure 1A, it is shown a chest X-ray (CXR) of patient 1 with irregular opacities and cavitations in the middle third and in right lung base. There was volumetric reduction of left lung with ipsilateral deviation of mediastinal structures, as well as thickening of pleura. In Figure 1B, it is shown a computed tomography of the thorax of patient 1 . The left lung was destroyed remaining only large cavitations. The right lung showed cavitations in the middle lobe, opacities and small nodules. In Figure 1C, the CXR of patient 2 showed volumetric reduction of left lung and interstitial-reticular opacities in both lungs, with left predominance, with cavity. There was a shift of mediastinal structures to left side. Patient 3 had an enormous caverna in upper left lobe, with thickening of right pleura and rough opacities and volumetric reduction of right lung (Figure 1D). Patient 4 presented interstitial opacities and nodules in both lungs (Figure 1E). Patient 5 (Figure 1F) had rough interstitial opacities in right lung base and discrete opacities in the right upper lobe.

\section{Discussion}

Multidrug-resistant Mycobacterium tuberculosis is an emerging and alarming health problem. The actual treatment regimens for MDR-TB are complex, expensive, long term, associated with high rates of side effects and poor outcome, and high morbidity and mortality [2-4,9,10,14-16]. Also, MDRTB is a complex and heterogeneous disease with multiple etiologic factors. The major cause for the problem is failure to adopt effective strategies for the control of the disease. Because an inadequate or poorly administered treatment regimen allows a drug-resistant strain to become a dominant strain $[5,6,17,18]$. In addition, poor adherence (or poor directly observed treatment - DOT), that increase resistance, is an important factor in this genesis. In addition to these factors, intolerance, side effects, poverty, social barriers, and poor medical management contribute as well to development of MDR-TB [5,19].

The current study documents that the main factors associated with MDR patients in Goiás were: poor adherence, drugs intolerance, and inaccuracy in the clinical management of patients. In this work gastric intolerance to anti-tuberculosis drugs was present in three individuals, and was neglected by medical services caring for the patients, and certainly contributed with poor adherence. Poverty and social barriers have been correlated to MDR, and one patient (patient \# 2) in this work was homeless. One (patient \# 5) had a contact with 
Figure 1. Radiological findings of MDR-TB patients. See details in the text
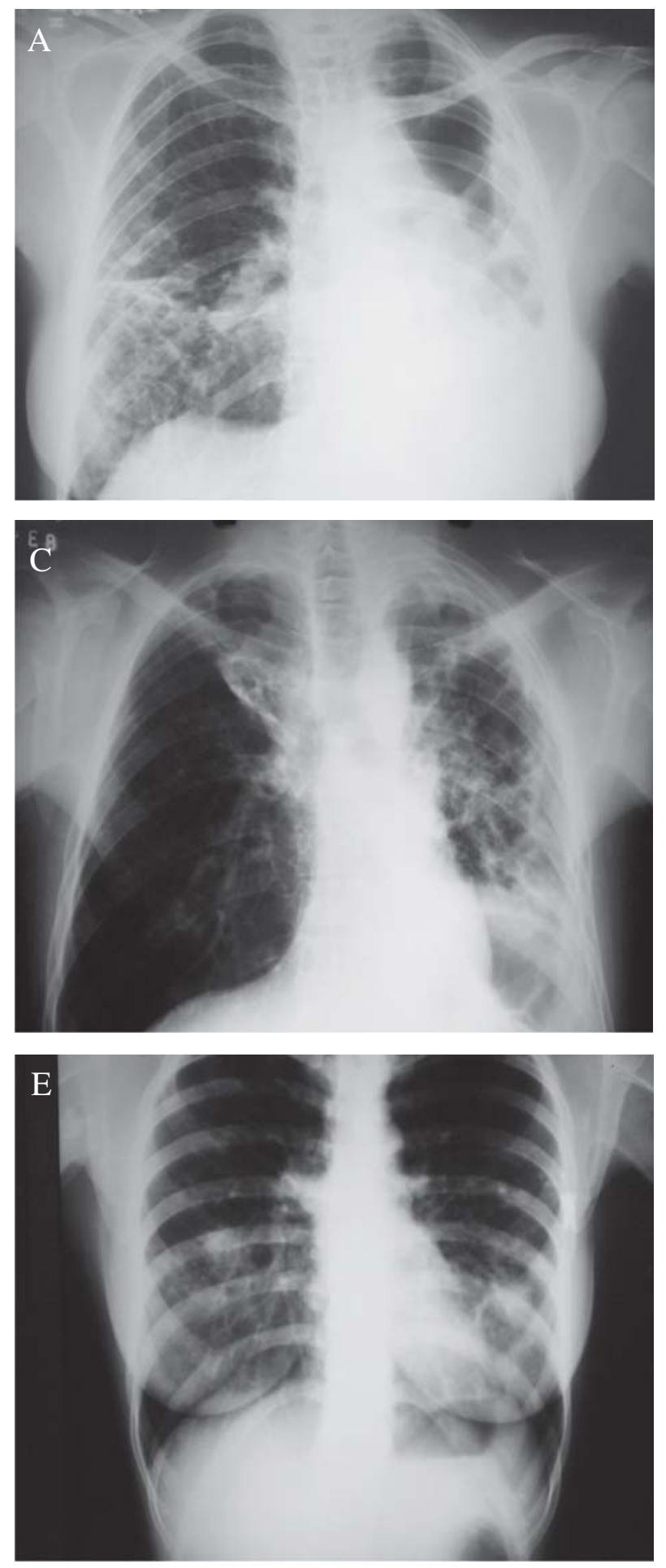

a culture-proven deceased case of MDR-TB, an important concern regarding MDR-TB. Our findings were similar with the ones from Barroso et al. [19] that also found that in 134 Brazilian MDR-TB patients, the factors associated to inadequate treatment leading to resistance were: non adherence, extreme poverty, drug intolerance, mistakes in
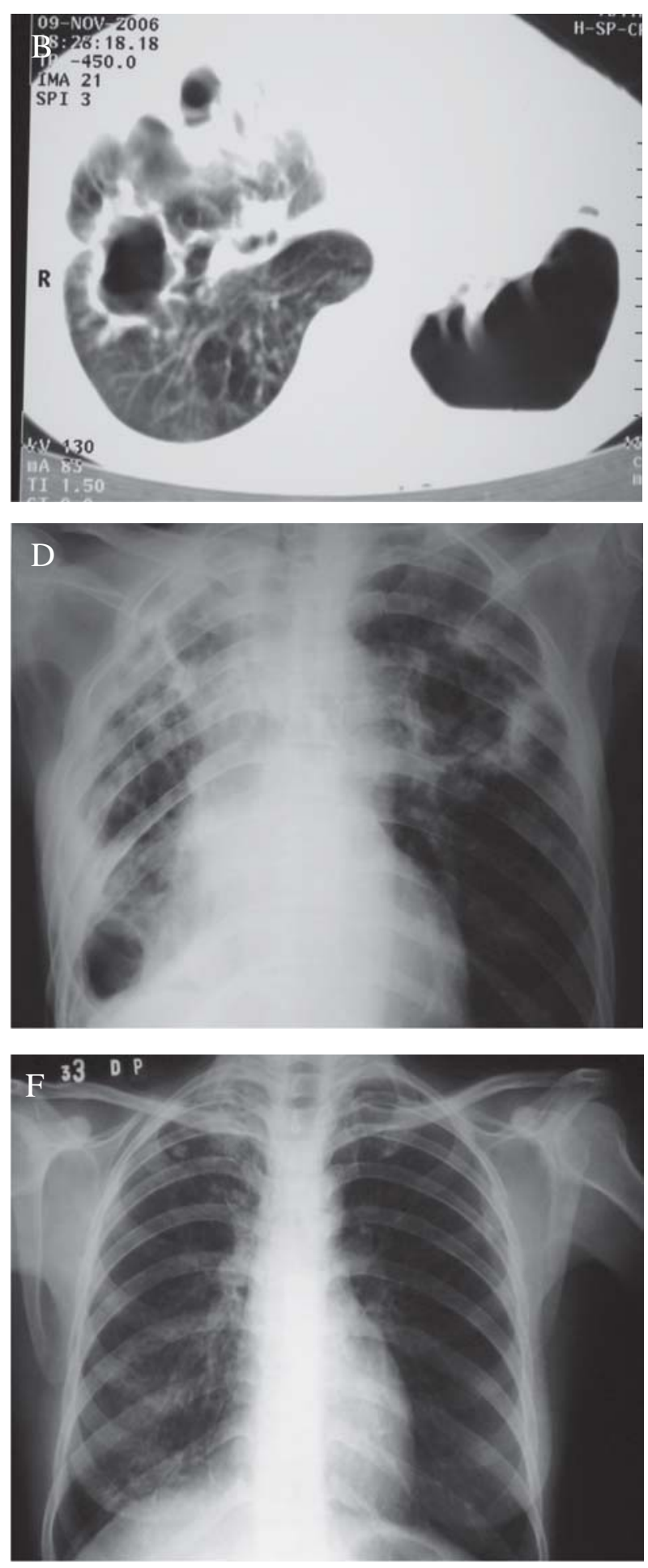

the clinical management, lack of medication, two or more treatments in the past, bilateral radiological lesions and large pulmonary cavitations.

The Brazilian Ministry of Health adopts a standardized treatment regimen for MDR-TB which includes AM, terizidone (TR), ofloxacin (OF), E, and Z during 18 months [6]. This regimen 
could not be adopted for the patients in our study because the patterns of sensibility and individual tolerance did not allow doing so. An injectable agent and a fluoroquinolone were always used, except in patient 1 that refused to use an injectable agent. The same patient refused to use $\mathrm{E}$ due to severe nausea and vomiting. The regimens containing fluoroquinolones like ofloxacin and levofloxacin are being related with better prognosis in MDR-TB patients [20]. Different treatment strategies can be adopted in MDR-TB cases. WHO recommends the following treatment strategies for MDR-TB: a) standardized treatment; the health service chooses the drugs according to the local Mycobacterium sensibility b) standardized treatment followed by individualized treatment; the regimen is adjusted when drug susceptibility testing (DST) becomes available; c) empirical treatment individualized based on patient history and then adjusted when DST results become available [5].

Ethionamide (ET) is in the group 4 of WHO antituberculosis drugs grouping, "oral bacteriostatic second-line anti-tuberculosis agents", and was used in patients 1,2 and 4 in order to compose an adequate regimen. Despite clarithromycin (CL) being in group 5, "anti-tuberculosis agents with unclear efficacy" (not recommended by WHO for routine use in MDR-TB patients), [5] it was used in patients 1, 3 and 5 in order to compose a satisfactory regimen.

In this work three patients had extensive and bilateral pulmonary disease. Barroso et al. [19] consider bilateral and extensive pulmonary cavities risk factors for MDR. In contrast, Chan et al. [11] did not find relationship between extensive lung lesions and poor outcome in MDR-TB patients. The extension of lung lesions could be related with the long course of disease in the patients in our study and not necessarily reflects major virulence. Kaplan et al. [21] demonstrated that the cavity surface is a microenvironment with failed immunity favoring drug resistance. In our study three patients presented lung cavities. The effect of mutational events that lead to antibiotic-resistant phenotypes may or may not have a predictable effect on the fitness of drug-resistant tuberculosis strains [18].

Surgical treatment has been considered an adjuvant therapy in the optimal management of MDR-TB cases, despite the absence of randomized trials to strengthen this issue. Sung at al. [22] and Chan et al. [11] consider that surgery increase the chance of cure in MDR-TB patients. Caminero [13] considers surgery when the patient meets the following conditions: a) localized lesions; b) adequate pulmonary functions; and c) lack of sufficiently available drugs to design a potent regimen. Lallo et al. [14], Furak et al. [23], Freixnet et al. [24], Shirashi et al. [25] and Takeda et al. [26] have a broader range of indications for pulmonary surgery in this setting, including high profile resistance, multiple previous relapses, persistent sputum positivity despite four-six months of therapy, high risk of recurrence based on the presence of destroyed lung tissue and cavities, hemoptysis, and drug allergy.

In this study only one patient (\# 3) underwent surgical procedure, a right upper lobe resection, in the tentative to improve the chance of cure because he had a localized disease during first MDR-TB treatment. In the postoperative period he developed bronchopleural fistula. He also was not able to take regularly the medications. In the beginning of his second treatment he had extensive bilateral disease and poor pulmonary function, which contraindicated surgical intervention. He also presented poor adherence and microbiological failure, despite the psychosocial support offered, dying six month after the beginning of re-treatment. Patient 1 and 2 had extensive and bilateral lesions precluding surgical procedures. Moreover patient 1 had poor pulmonary function. Patients 4 and 5 did not have extensive disease and were adherent to the treatment.

The obit in the present series occurred in the patient with more prolonged course of TB. His first diagnosis was done in 1999. His first course of MDR-TB regimen was initiated in 2000. He was the only patient in whom a surgical approach was done, but the obit was not related to it. He had a TST of $30.0 \mathrm{~mm}$, which could be connected to higher tissue damage.

MDR-TB is more prevalent in patients co-infected with HIV, and is associated with high mortality [14-16, 27] but in our study all patients were HIV negative. Goiás is a state with a medium rate of incidence of HIV/AIDS (13.1/100,000 in 2005) [28]. In 2006, $10.9 \%$ of AIDS patients developed tuberculosis in Goiás, and $5.1 \%$ of new cases of tuberculosis were HIV positive [29]. HIV+ co-infected MDR-TB patients could exist in Goiás, but not diagnosed and/or not referred to our service.

The DOTS strategy (directly observed treatment, short course, DOTS strategy) is strongly recommended to face TB epidemic and MDR-TB emergence. The DOTS strategy includes five elements: a) political commitment with increased and sustained financing; b) case detection through quality-assured bacteriology; c) standardized treatment with supervision (DOT - direct observed treatment) and patient support; d) an effective drug supply and management system; and e) monitoring and evaluation system, and impact measurement [3,5].

The state of Goiás health system has not universally implemented DOTS programs, a well-known factor related to drug resistance. Certainly the adoption of DOTS will help to prevent the occurrence of new MDR-TB cases, and the supervised treatment of the MDR-TB active cases could improve the chances of cure.

Associated with the efforts to universally adopt DOTS, there is a need to develop new drugs to face this scourge [30-32]. The use of adjuvant therapies, like the use of interferon-J (IFN-J) in MDR-TB cases, has yielded contradictory results. Mendez et al. [33] found better prognosis in MDR-TB patients using IFN-J. However, IFN-J was associated with high level of side effects and no benefits were found in the work of Park et al. [34].

Palmero et al. [12] found that hospital admission was related with treatment success as well as Chan et al. [11]. Based on this observation, Griffith [35] argued if MDR-TB patients should not be treated at a hospital. The adoption of this strategy certainly will raise the costs of the treatment, already high. 
Some M. tuberculosis strains, like Beijing/W genotype, is associated with drug resistance [36] reinforcing the needs of studies in this field in our region and we are currently doing molecular analysis of the M. tuberculosis strains isolated from these patients.

In our opinion it is important that the Brazilian Ministry of Health adopts the WHO definition of MDR-TB case. Certainly with the Brazilian definition there is a great possibility that the MDR problem is being underestimated in Brazil. It is also important because only patients that fulfill the Brazilian definitions will have access to second line drugs provided by the Ministry of Health. It is essential to know the profile of MDR-TB patients in different regions from Brazil due to diverse local realities, concerning socioecomonic aspects and programs for tuberculosis control. We believe that further studies focusing immunological aspects and genotyping of the MDR M. tuberculosis strains will add more important clues to our current understanding of this disease.

The MDR-TB cases related in this work were associated with poor individual management and/or lack of an effective TB program, especially concerning supervised treatment and case vigilance.

\section{References}

1. Frieden T.R., Sterling T.R., Munsiff S.S., et al. Tuberculosis. Lancet 2003;362:887-99.

2. Meya D.B., McAdam K.P.W.J. The TB pandemic: an old problem seeking new solutions. J Inter Med 2004;261:309-29.

3. World Health Organization. WHO REPORT 2007: Global Tuberculosis Control: Surveillance, Planning, Financing. Geneve; 2007.

4. Pablos-Mendez A., Raviglione M.C., Laszlo A., et al. Global surveillance for antituberculosis-drugs resitance, 1994-1997. N Engl J Med 1998;338(23):1641-9.

5. World Health Organization. Guidelines for the programmatic management of drug-resistant tuberculosis. Geneva: World Health Organization; 2006.

6. Ministério da Saúde. Tuberculose multirresistente - Guia de vigilância epidemiológica. Rio de Janeiro: Ministério da Saúde; 2006.

7. World Health Organization. Case definition for extensively drugresistant tuberculosis. Wkly Epidemiol Rec 2006;81(42):408.

8. Center for Diseases Control and Prevention. Emergence of Mycobacterium tuberculosis with Extensive Resistance to Second-Line Drugs - Worldwide, 2000-2004. Morb Mortal Wkly Rep 2006;55.

9. Flament-Saillour M., Robert J., Jarlier V., Grosset J. Outcome of multi-drug-resistant tuberculosis in France: a nationwide casecontrol study. Am J Respir Crit Care Med 1999;160(2):587-93.

10. Drobniewski F., Eltringham I., Graham C., et al. A national study of clinical and laboratory factors affecting the survival of patients with multiple drug resistant tuberculosis in the UK. Thorax 2002;57:810-6.

11. Chan E.D., Laurel V., Strand M.J., et al. Treatment and outcome analysis of 205 patients with multidrug-resistant tuberculosis. Am J Resp Crit Care Med 2004;169:1103-9.

12. Palmero D.J., Ambroggi M., Brea A., et al. Treatment and followup of HIV-negative multidrug-resistant tuberculosis patients in an infectious diseases reference hospital, Buenos Aires, Argentina. Int J Tuberc Lung Dis 2004;8(6):778-84.

13. Caminero J.A. Treatment of multidrug-resistant tuberculosis: evidence and controversies. Int $\mathrm{J}$ Tuberc Lung Dis 2006; $10(8): 829-37$.
14. Lalloo U.G., Naidoo R., Ambaran A. Recente advances in the medical and surgical treatment of multi-drug resistant tuberculosis. Curr Opin Pulm Med 2006;12:179-85.

15. Kang Y.A., Choi Y.-J., Lee S.M., et al. Cost of treatment for multidrug-resistant tuberculosis in South Korea. Respirology 2006; $11: 793-8$.

16. Resh S.C., Salomon J.A., Murray M., Weinstein M.C. Costeffectiveness of treating multidrug-resistant tuberculosis. PLoS Med 2006;3(7):1048-57.

17. Rattan A., Kalia A., Ahmad N. Multidrug-resistant Mycobacterium tuberculosis: molecular perspectives. Emerg Infect Dis 1998;4(2): 195-209.

18. Cohen T., Sommers B., Murray M. The effect of drug resistance on the fitness of Mycobacterium tuberculosis. Lancet Infect Dis 2003;3:13-21.

19. Barroso E.C., Mota R.M.S., Morais M.F.M., et al. Fatores associados aos tratamentos inadequados em portadores de tuberculose multirresistente. J Pneumo 2003;29(6):350-7.

20. Yew W.W., Chan C.K., Chau C.H., et al. Outcomes of patients with multidrug-resistant pulmonary tuberculosis treated with ofloxacin/levofloxacin-containing regimens. Chest 2000; $117(3): 744-51$.

21. Kaplan G., Post F.A., Moreira A.L., et al. Mycobacterium tuberculosis growth at the cavity surface: a microenvironment with failed immunity. Infect Immun 2003;71(12):7099-108.

22. Sung S.W., Kang C.H., Kim Y.T., et al. Surgery increased the chance of cure in multi-drug resistant pulmonary tuberculosis. Eur J Cardiothorac Surg 1999;16(2):187-93.

23. Furak J., Trojan I., Szoke T., et al. Surgical intervention for pulmonary tuberculosis: analysis of indications and perioperative data relating to diagnostic and therapeutic resections. Eur J Cardiothorac Surg 2001;20(4):722-7.

24. Freixinet J., Rivas J.J., Castro F.R., et al. Role of surgery in pulmonary tuberculosis. Med Sci Monit 2002;8(12):782-6.

25. Shiraishi Y., Nakajima Y., Katsuragi N., et al. Resectional surgery combined with chemoteraphy remains the treatment of choice for multidrug-resistant tuberculosis. J Thorac Cardiovasc Surg 2004; $128: 523-8$.

26. Takeda S., Maeda H., Hayakawa M., et al. Current surgical intervention for pulmonary tuberculosis. Ann Thorac Surg $2005 ; 79(3): 959-63$.

27. Patrice J., Severe P., Ferdinad S., et al. Multidrug-resistant tuberculosis at an HIV center in Haiti. AIDS 2006;20(3):415-8.

28. Ministério da Saúde. Boletim Epidemiológico - AIDS-DST, ano III, no. 1, Ministério da Saúde; 2006.

29. Secretaria de Estado da Saúde, Estado de Goiás. http://www.saude. go.gov.br/index.php?idMateria=18703. Accessed in 21/05/2007.

30. O'Brien R.J., Nunn P.P. The need for new drugs against tuberculosis. Obstacles, opportunities, and next steps. Am J Resp Crit Care Med 2001; $162: 1055-8$.

31. Duncan K., Barry III C.E. Prospects for new antitubercular drugs. Curr Opin in Microbiol 2004;7:460-5.

32. Souza M.V.N., Vasconcelos T.R.A. Fármacos no combate á tuberculose: passado, presente e futuro. Química Nova 2005;28(4):678-82.

33. Mendez R.S., Garcia I.G., Olivera N.F., et al. Adjuvant interferon gamma in patients with drug - resistant pulmonary tuberculosis: a pilot study. BMC Infect Dis 2004;4(44):1-8.

34. Park S.-K., Cho S., Lee I.-H., et al. Subcutaneously administered interferon-gamma for treatment of multidrug-resistant pulmonary tuberculosis. Int J Infect Dis 2007;in press.

35. Griffith D.E. Treatment of multidrug-resistant tuberculosis. Should you try this at home? Am J Resp Crit Care Med 2004;169:1082-3.

36. European Concerted Action on New Generation Genetic Markers and Techniques for the Epidemiology and Control of Tuberculosis. Beijing/W genotype Mycobacterium tuberculosis and drug resistance. Emerg Infect Dis 2006;12(5):736-43. 